\title{
Socially relevant philosophy of science: an introduction
}

\author{
Carla Fehr • Kathryn S. Plaisance
}

Received: 22 November 2010 / Accepted: 23 November 2010 / Published online: 7 December 2010

(C) Springer Science+Business Media B.V. 2010

\begin{abstract}
This paper provides an argument for a more socially relevant philosophy of science (SRPOS). Our aims in this paper are to characterize this body of work in philosophy of science, to argue for its importance, and to demonstrate that there are significant opportunities for philosophy of science to engage with and support this type of research. The impetus of this project was a keen sense of missed opportunities for philosophy of science to have a broader social impact. We illustrate various ways in which SRPOS can provide social benefits, as well as benefits to scientific practice and philosophy itself. Also, SRPOS is consistent with some historical and contemporary goals of philosophy of science. We're calling for an expansion of philosophy of science to include more of this type of work. In order to support this expansion, we characterize philosophy of science as an epistemic community and examine the culture and practices of philosophy of science that can help or hinder research in this area.
\end{abstract}

Keywords Philosophy of science - Socially relevant philosophy of science · Epistemic communities · Social epistemology

C. Fehr $(\bowtie)$

Department of Philosophy and Religious Studies, Iowa State University, 402 Catt Hall, Ames,

IA, 50011, USA

e-mail: cfehr@iastate.edu

K. S. Plaisance $(\varangle)$

Centre for Knowledge Integration, University of Waterloo, 200 University Avenue West, Waterloo, ON, N2L 3G1, Canada

e-mail: kplaisan@uwaterloo.ca 


\section{Introduction}

This project arose out of a keen sense of lost opportunities for philosophy of science to effectively contribute to public welfare. We see opportunities for excellent work in philosophy of science to have a greater impact on the practices and products of scientific research. We also see opportunities for philosophers of science to aid scientists in developing policy relevant research that is both epistemically rigorous and sensitive to the ethical and political needs of a wider range of publics both locally and globally. We call this body of work socially relevant philosophy of science (SRPOS). As the papers in this volume indicate, while SRPOS can aid in scientific practice and facilitate effective engagement between science and the publics it serves, it also can aid in the critical assessment of traditional topics within philosophy of science and fruitfully direct the development of new questions and approaches within philosophy of science itself.

This special issue arises out of a conference and workshop intended to address how philosophy of science might better take advantage of these opportunities. The 2008 American Philosophical Association Pacific Division mini-conference on "Making Philosophy of Science More Socially Relevant" focused on philosophical analyses of scientific research on topics of social, ethical, and political significance. The 2008 Philosophy of Science Association workshop, "How Philosophers of Science Can Take Up Socially Relevant Roles", focused on the ways that disciplinary practices and reward structures within philosophy of science hinder the development of SRPOS. We are grateful for the fruitful discussions at these events, which demonstrated a lively interest in ways that philosophy of science could be more socially relevant.

SRPOS is a highly pluralistic endeavor. It includes philosophical engagement with scientific research on socially relevant topics, philosophical activities that attend to the interactions among scientists and various communities that contribute to and are affected by scientific research, as well as philosophy of science disseminated in ways that reach beyond communities of philosophers.

In Sect. 2 we answer the question, 'What is SRPOS?' We explain the notion of SRPOS and show how the papers in this volume demonstrate the plurality of facets and themes apparent in this body of work. In Sect. 3 we consider why SRPOS is important. We demonstrate that SRPOS has significant social, scientific, and philosophical benefits. And in Sect. 4, we consider why SRPOS is something in which philosophers of science, qua philosophers of science, ought to engage. Finally, in Sect. 5, we consider possible barriers to the development of more SRPOS in philosophy of science and suggest that one way to respond to those barriers is to think about philosophy of science itself as an epistemic community.

\section{SRPOS: a pluralistic endeavor}

SRPOS is a highly pluralistic endeavor. We see at least three facets of this kind of philosophical work. First, SRPOS focuses on philosophical analyses of scientific research topics and scientific practices that are directly relevant to public welfare, such as investigations of race and genomics, biomedical research, or special interest science. This 
can involve philosophical investigations of policy relevant science, including science that has the capacity to harm marginalized or otherwise vulnerable groups of people, or science that has the capacity to benefit central and powerful groups of people while those benefits are withheld from members of marginalized groups. As illustrated in Sect. 3, these philosophical analyses of socially relevant topics can include a range of philosophical approaches, where some SRPOS uses traditional methods or approaches in philosophy of science, such as standard conceptual analysis, to address socially relevant science; some engages socially relevant scientific topics in ways that allow for the assessment or advancement of traditional topics in philosophy of science (e.g., prediction or explanation); and some focuses on socially relevant science to develop new approaches to and positions within philosophy of science.

Second, as a result of being concerned with scientific practices relevant to public welfare, SRPOS can also focus on or engage various stakeholder groups. These groups can include policy makers, various publics, disadvantaged or marginalized populations, or even scientific practitioners themselves. SRPOS often considers the fairness of the distribution of benefits and harms of scientific research with respect to various stakeholders. Importantly, the influences of scientific knowledge and scientific knowledge production practices on these various groups can be, and often are, reciprocal. Just as stakeholders can benefit from or be harmed by scientific practice, members of these groups can also have an influence on the successes or failures of scientific practice, both in terms of knowledge production and knowledge sharing. Some work in SRPOS examines the reciprocal nature of this relationship, and some concentrates on groups of people who are likely to be most seriously affected by particular scientific knowledge claims. Examples of the former include examinations of trust between scientific and lay communities, as illustrated in this issue. Examples of the latter include philosophical analyses demonstrating that climate change may most adversely affect people from poor countries, that environmental toxins may disproportionately hurt children, pregnant women and those in low-income neighborhoods, that research on gender differences may harm women by reinforcing harmful gender inequities, and that research on biology and race can lead to policies that exacerbate racial injustices and harm people of color. In many cases, SRPOS not only investigates scientific research that is socially relevant, but also focuses specifically on the perspectives and interests of those who are most likely to be harmed by the results of such research.

A third aspect of SRPOS concerns the practices and venues in which philosophers engage in order to maximize the social impact of their work. For example, some philosophers engage in collaborative relationships with educators, policy makers, or scientists, and disseminate their work in venues where it is likely to transcend the disciplinary boundaries of philosophy and impact scientific practice and public policy. Collaborative relationships can help philosophers to produce work that better meets the needs of scientists, policy makers, and the various publics affected by scientific research, and can inform their own philosophical work as well. Disseminating philosophical work beyond the boundaries of philosophy can put it in the hands of people who are in a position to influence policy and scientific practice, and in some cases philosophers of science can themselves influence policy and scientific practice.

The papers in this volume exemplify varying combinations of these facets of SRPOS. While there are many ways to conduct SRPOS, there are four general themes 
represented by the authors in this volume. These include: (1) developing collaborative relationships with scientists, (2) addressing policy, regulation, and institutional structure, (3) investigating the relationships among scientific and non-scientific communities, and (4) assessing the effectiveness of the disciplinary norms, practices, and structures of philosophy of science itself. While each of these themes can be distinguished conceptually, in most cases these activities are intertwined. For instance, improving the institutional structure of biomedical research programs could be facilitated by developing collaborative relationships with scientific practitioners. Likewise, examining and improving the disciplinary practices and reward structures of philosophy of science might be necessary to allow philosophers to do SRPOS (including (1), (2), and (3), above) without retarding their career advancement, an issue that we address in detail in Sect. 5.

\subsection{Collaboration with scientists}

Heather Douglas argues that to do SRPOS, we need to engage with scientists and users of science "on the ground," rather than importing philosophical ideas "off-the-shelf." This means that rather than simply applying philosophical theories to scientific case studies, philosophers are well served by directly engaging scientists. Engagement allows philosophers to address issues that arise from a scientific perspective in ways that benefit scientific practice and facilitate the advancement of philosophy of science. For example, Douglas shows that that the value-free ideal of science fails to provide normative guidance for scientists involved in risk analysis. Engaging with scientists "on the ground" facilitated her development of a normative model of the constructive role of values in scientific practice, an advancement for philosophy of science and a useful tool for scientists.

Nancy Tuana also argues for engagement, specifically for an embedded philosophy of science in which philosophers partner with scientists in the classroom as well as on the research team. In particular, she draws on the strengths and limitations of bioethics to develop a model, the Ethical Dimensions of Scientific Research, that highlights the need for understanding the interactions among ethical and epistemic factors in scientific practice, an understanding that philosophers of science are in an excellent position to contribute. Tuana exemplifies this embedded approach in her own collaborative relationships with climate scientists and national and international climate policy makers, which leads to another theme: philosophy of science that addresses policy, regulation, and institutional structure.

\subsection{Addressing policy, regulation, and institutional structure}

Tuana argues for, and exemplifies through her own work, the usefulness of uniting philosophy of science, epistemology, and ethics in the service of developing effective science-based policy. Tuana shows how social values play a role in the construction of climate models, and in turn how these models can be used to support policies that may result in the disproportionate harm of citizens of poor countries. Her analyses reveal that scientific knowledge informed by a particular set of values can create this risk of 
harm, a finding that is important to communicate effectively to relevant policy makers. Tuana has been involved with United Nations climate change conferences, and as director of the Rock Ethics Institute she developed the Collaborative Program on the Ethical Dimensions of Climate Change, an international group of scholars and institutions who work to ensure that climate policies, ranging from local to international, are "well rooted in the principles of justice". 1

Julian Reiss identifies epistemic and ethical failures arising out of the institutional structure of biomedical research (BMR). He demonstrates that BMR is not as innovative as many believe and, perhaps more importantly, it creates and perpetuates social injustices by focusing on chronic diseases suffered by relatively few wealthy people as opposed to focusing on decreasing the global disease burden. Reiss argues that these problems arise out of regulatory failures and the institutional structure of BMR. As he points out, philosophy of science, with its recent focus on scientific practice and the epistemic structure of knowledge-producing institutions, is in an excellent position to address these issues. Reiss develops a proposal for organizing and regulating biomedical research such that it better supports global and national public welfare.

Kristin Shrader-Frechette argues that philosophy of science can be made more socially relevant by doing standard conceptual analysis with respect to special-interest science (SIS). Shrader-Frechette illustrates this with a detailed example of "hormesis" (according to which low levels of toxins can emit positive effects), showing how conceptual obfuscation, methodological flaws, and faulty inferences have led to inadequate regulatory policy, as well as conclusions used by pretrial judges to deny victims of low-dose toxins jury trials. Shrader-Frechette also notes that there is a significant amount of uncertainty in SIS, which leaves space for ethical and policy judgments to enter, and argues that defenders of hormesis have overlooked important ethical issues. Her work illustrates a socially relevant role for philosophers of science who use traditional methods (e.g., conceptual analysis, evaluation of methodology and inference-making, and identifying the role of values in science), on topics directly relevant to public welfare.

The need for collaboration and broader dissemination of this work is clear, especially when the goals of the analyses include changes to institutional structures or public policy. Shrader-Frechette explicitly argues that more philosophers of science should disseminate their work to venues where it is likely to have a direct impact on the public good. Her own work has been given uptake in science and policy journals, which has led to invitations to join important science-policy groups, such as the US National Academy of Sciences, regulatory committees of the US Department of Energy, and the US Environmental Protection Agency Science Advisory Board. ${ }^{2}$ Shrader-Frechette's research career exemplifies a balance between traditional work in philosophy of science and active engagement with policy makers.

Through collaborations with scientists and engagement with policy makers, several of the authors in this volume not only argue for improving scientific practice so that it more effectively contributes to public welfare, but they develop relationships with

\footnotetext{
1 See http://rockethics.psu.edu/climate/.

2 See http://www.nd.edu/ kshrader/policy/.
} 
scientists and policy makers who are in social positions to actually make these changes. This engagement with 'change makers' includes both collaborative interactions and presenting philosophical work in venues where it is likely to reach a significant audience beyond tightly construed philosophical communities.

\subsection{Investigating intercommunity relations}

Kyle Whyte and Robert Crease, as well as Heidi Grasswick, offer more general considerations of the role of trust between scientific and lay communities. Whyte and Crease point out that when trust is lacking between scientists and lay public(s), scientists suffer because they are less likely to benefit from local knowledge and lay publics suffer because they are less likely to reap the benefits of scientific research. They explore this problem by considering cases where trust fails and where it succeeds. Trust can fail when scientists do not consider the scientifically relevant expertise of stakeholder groups, so Whyte and Crease call for the development of notions of expertise that respect local knowledge. They also show how trust can succeed by pointing to a case of scientific research that better meets its own goals as a result of transparency and collaboration between scientists and the relevant publics.

Heidi Grasswick argues that scientific communities require the trust of lay communities in order to meet their constitutive goals of producing objective knowledge and knowledge that can be put to use. She looks at scientific practices that can rationally ground the trust of members of lay communities. In particular, Grasswick uses cases of scientific "knowledge-sharing whistle blowers" to demonstrate the existence of public expectations that scientists share knowledge with members of lay communities and to show that failures to share relevant and significant knowledge, especially when such failures can cause harm, erodes public trust in science. Grasswick argues that this erosion of trust is a particularly significant issue with respect to members of marginalized communities who may already have reason not to trust scientific authority.

Both of these papers, with their focus on the relationship between science and society, highlight the practical, ethical, and epistemic significance of attending to the relationships between scientific communities and various stakeholder groups.

\subsection{Changing philosophical practice}

While all of the papers in this issue are calls to expand philosophy of science in order to make our work more socially relevant, and indeed more socially engaged, Richardson and Gannett specifically focus on features of philosophy of science that impede efforts to conduct SRPOS and philosophy of science more generally.

Lisa Gannett evaluates philosophical research concerning biology (in particular genomics) and the 'reality' of various concepts of race, pointing out that philosophers of science tend to approach questions of biology and race as a natural kinds problem. She argues that using traditional notions of natural kinds to investigate and evaluate race concepts leads philosophers to overlook questions of social and political import, and can even foreclose such questions altogether. As she illustrates, these approaches tend to dichotomize race as being either biologically real or socially constructed, which does 
not allow for interactions between biological and social factors. She argues that philosophers of science need to be open to examining causal interactions that cross multiple levels of analysis, as well as examining the contexts in which genomics research is used and developed. This, in turn, makes room for considerations of the political and ethical ramifications of the various ways of characterizing racial differences.

While Gannett focuses on the ways that traditional notions of natural kinds limit philosophers' analyses of race concepts, Sarah Richardson focuses on ways that disciplinary norms and practices in philosophy of science can hinder SRPOS.

Richardson provides a rich history of the development of feminist philosophy of science, showing how it is related to academic feminism more broadly, focusing on its interdisciplinarity and highlighting significant contributions to SRPOS made by feminist philosophers of science. These contributions include diagnosing how gender bias has played and continues to play a role in scientific practices and theories, and more general contributions with respect to the role of values in scientific research. Richardson argues that some disciplinary practices within philosophy of science create barriers for feminist philosophy of science. She documents ways that feminist philosophy of science is often marginalized by and misrepresented in philosophy of science discourse. She also argues that philosophy of science pushes feminist work on bias removal at the expense of more philosophically fruitful work on developing ethical-epistemic models of the role of values in scientific practice. In Sect. 5, we return to a discussion of the ways that the disciplinary reward structure in philosophy of science can either help or hinder SRPOS.

The four themes running through the papers in this volume represent a diversity of ways of doing SRPOS. What the papers all have in common is that they exemplify one or more of the facets of SRPOS - engaging topics of socially relevant science, doing so in a way that attends to a variety of stakeholders (such as practicing scientists, lay communities, and policy makers, as well as the interactions among them), and addressing how this work can be broadly disseminated - all of which is motivated by a concern for public welfare.

\section{Social, scientific, and philosophical benefits of SRPOS}

SRPOS engages socially relevant science, that is, scientific practices or products that can improve or harm public welfare. This can translate into social benefits for policy makers or for the public(s). There is also a wide range of ways that SRPOS benefits science-by supporting or contributing to the epistemic and ethical excellence of scientific research on policy relevant topics, or on socially relevant topics more generally. Finally, SRPOS can benefit mainstream philosophy of science (POS), either by advancing mainstream approaches or concepts in POS, or by developing new questions, approaches, and insights.

\subsection{Social benefits of SRPOS}

There are many pressing and thorny social issues that scientific research is wellpositioned to ameliorate or exacerbate, such as those that threaten the welfare of 
humans, animals, or the environment. One way that philosophy of science can have a positive impact is by conducting philosophical analyses on socially relevant science. ${ }^{3}$ This might include, for example, clarifying key concepts in socially relevant science, identifying questionable methodological assumptions, pointing to epistemic failures and suggesting improvements, or determining epistemic reasons for why potentially useful scientific knowledge is not being given uptake. This work can provide social benefits to a wide range of groups, including policy makers, lay communities, and others. For example, Shrader-Frechette's work on environmental toxins is directly relevant to policy makers and courtroom judges, and, by way of the decisions they make, impacts victims of environmental injustice.

One way that SRPOS can translate into these social benefits is by being made accessible to those individuals or communities that are in a position to make use of the products of philosophy of science. As we indicated in Sect. 1, one of the main motivations behind this project was what we saw as missed opportunities for work in philosophy of science to contribute such benefits. Oftentimes, SRPOS may be socially relevant but not sufficiently engaged with the wider communities to whom this research is most relevant. Thus, to reap the social benefits of SRPOS, it is useful to broaden philosophy of science research practices to include collaboration with and dissemination to broader audiences. ${ }^{4}$

\subsection{Scientific benefits of SRPOS}

SRPOS can also benefit scientific practice in a variety of ways, which can involve constructive collaboration with scientists as well as criticism of scientific practices. ${ }^{5}$ Along these lines, Reiss identifies ethical and epistemic failures with biomedical research, offering solutions that would not only lead to a more just distribution of research efforts, but improved scientific knowledge as well. SRPOS can help to identify problems not only with the production of scientific knowledge, but also with its uptake. This is illustrated nicely by the papers on trust between science and society: Whyte and Crease show how, in some cases, scientists could better make use of scientifically-relevant knowledge by broadening their conception of expertise, and Grasswick identifies failures in knowledge-sharing practices that translate into a lack of public uptake of scientific knowledge (which is especially problematic for areas of science that have as constitutive goals the application and use of their research products). These benefits arise from philosophical work but will have an impact only if they reach the relevant communities, suggesting that wider dissemination practices can be an important part of SRPOS.

\footnotetext{
3 See Kourany (2003a,b); Giere (2003) —an interesting exchange on the social benefits of feminist philosophy of science.

4 Both Shrader-Frechette and Tuana are excellent examples of this, as both of them not only publish their work in wider venues, but also serve as a science policy advisors, thus working in direct contact with those to whom their work is relevant.

5 We do not wish to preclude SRPOS that offers a more radical criticism of scientific practice or assumptions (see, for example, Fuller 2008; Keller 1996).
} 


\subsection{Philosophical benefits of SRPOS}

In addition to having positive impacts on wider communities, SRPOS also provides benefits to POS itself. By collaborating with scientists and other stakeholders, and engaging with social issues, SRPOS can develop new areas of philosophical research, raise questions that are interesting and philosophically relevant, and offer new insights on traditional topics. The first of these is exemplified by Whyte and Crease's, and Grasswick's, work on trust between science and society, which builds on previous analyses of trust as an epistemic concept. These previous analyses examine the role of trust within knowledge-producing communities, while SRPOS considers a wider set of communities and examines knowledge sharing practices in relation to knowledge production, an advance in social epistemology. SRPOS can also lead to new and potentially fruitful philosophical questions, as illustrated by Gannett's argument that the standard natural kinds approach to race forecloses certain questions. By attending to local contexts, as well as interactions between biological and social factors, she identifies new questions related to research in race and genomics that would benefit from analysis. More generally, SRPOS supports a growing body of work, exemplified by much feminist philosophy of science, on the interactions between ethical and epistemic aspects of scientific practice and knowledge production. Finally, as Douglas argues, SRPOS can bring important insights to philosophical topics, such as prediction, explanation, and weight of evidence.

These benefits—-social, scientific, and philosophical—are sometimes interdependent and often mutually reinforcing. For example, the social benefits of SRPOS often result from identifying ways in which scientific practices can be improved, while many philosophical benefits arise either from fruitful collaborations with scientific practitioners or by attending to issues of social import. Tuana's work on ethical-epistemic issues in climate change modeling is a particularly nice example of how SRPOS can simultaneously benefit several communities. This work consists of examining underlying assumptions and values in climate change models_-models that include a significant amount of uncertainty - and the policy implications of decisions that practitioners must make in the face of such uncertainty. These analyses can aid scientists by making explicit how value choices influence their modeling activities and results, which can in turn aid policy makers. In addition, by examining real-world, complex scientific issues at the interface of ethics and epistemology, SRPOS can address philosophical questions that do not fall neatly within the purview of either mainstream philosophy of science or bioethics. As Tuana's work illustrates, some of the philosophical benefits that SRPOS offers are a direct result of taking a socially and scientifically engaged approach.

Lastly, in an economic climate in which university programs are shrinking, it can be useful to emphasize that philosophy, in addition to its intrinsic value in terms of knowledge production, can contribute in more concrete ways to the public good. Furthermore, as we argue in the next section, philosophers of science are well positioned to do work that provides the types of benefits discussed above, and this work-i.e., SRPOS - is consistent with the historical goals of our discipline, as well as with contemporary research that takes a feminist or naturalistic approach. 


\section{Situating SRPOS in philosophy of science}

There are several reasons why the work we're calling for in this issue - that is, work that is scientifically and socially engaged and is concerned with public welfare (i.e., SRPOS) - ought to fall within the purview of philosophy of science, and should be valued and supported by our discipline, including not only POS but philosophy more generally. First, philosophers of science have training, skill sets, and disciplinary locations that make us particularly well suited to analyzing scientific practice and serving as effective science critics. Second, with its focus on socially relevant topics, its openness to considerations of the interactions among epistemology and ethics, and its engagement with a wider range of stakeholder groups, SRPOS is consistent with some significant historical and contemporary practices in POS. (However, many of these practices have been largely marginalized or undervalued, which is unfortunate given the wide range of benefits associated with SRPOS.) Finally, as some have argued, philosophers of science — or, better yet, philosophy of science-may have a responsibility to do work that can positively impact public welfare.

4.1 Philosophers of science are well positioned to analyze socially relevant scientific practice

As philosophers of science, we have particular skill sets that allow us to carefully analyze the practices and products of science. These include conceptual, methodological, and inferential analysis; revealing underlying assumptions or values in scientific reasoning; providing epistemic tools for scientific practitioners (e.g., weight of evidence approaches, conceptions of expertise, facilitating trust through knowledge sharing); and evaluating ways in which scientific knowledge is translated, used, or applied. One way to make our work more socially relevant is to apply this standard philosophical skill set to scientific practices that have a direct impact on public welfare.

In addition to these skill sets, much of the work in SRPOS requires philosophers of science to collaborate with practicing scientists or to analyze the ethical issues that are often intertwined with epistemic ones (e.g., examining the role of values in scientific decision-making in the face of uncertainty). The former is supported by training in naturalistic approaches and the ability to readily cross between philosophical and scientific domains. This is something that many philosophers of science already have the necessary education and training to do, though it is important to recognize the time and effort that such philosophers must devote to being able to do this well. The latter-analyzing ethical issues that are intertwined with epistemic ones - is supported by expertise in ethics, something that many philosophers have, at least to some extent. As many of the authors point out, many of the methodological and inferential choices that scientists make involve uncertainty and are value laden, and hence require analyses at the interface of ethics and epistemology. Unfortunately, such analyses often fall through the cracks of mainstream POS and bioethics (see especially Douglas, Reiss, and Tuana). Finally, philosophers are in a good disciplinary location for acting as science critics, since, as Shrader-Frechette points out, we typically have no financial conflicts of interest unlike scientists whose work is funded by special interest groups. 


\subsection{SRPOS is consistent with the history of philosophy of science}

Recent work on the history of philosophy of science suggests that, prior to WWII, one of the constitutive goals of the discipline was to attend to social issues and examine science in its social context. ${ }^{6}$ Douglas specifically situates SRPOS in the history of POS, pointing out that, although philosophy of science currently focuses on the epistemology and metaphysics of science, the discipline was not always so narrowly construed: "According to the PSA by-laws first developed in 1946, the fledgling society was dedicated to "furthering of the study and discussion of the subject of philosophy of science, broadly interpreted, and the encouragement of practical consequences which may flow therefrom of benefit to scientists and philosophers in particular and to men of good will in general.' (Philosophy of Science, 1948, vol. 15, p. 176). It seemed obvious to the early PSA members that there should be such 'practical consequences ... of benefit' to society" (Douglas, this issue). In other words, during the early history of the PSA, the association not only included SRPOS, but also considered social relevance to be one of its goals.

4.3 SRPOS is consistent with some contemporary practices in philosophy of science

Insofar as SRPOS involves philosophers engaging with scientists, it is consistent with a naturalist tradition within philosophy of science. For example, to develop a deep understanding of the science they study, it is fairly commonplace for philosophers of biology to develop some sort of collaborative relationship with practicing scientists. Moreover, some philosophers of science have collaborated with scientists, made their analyses accessible to them, and have successfully informed scientific practice. As Shrader-Frechette points out: "Ernst Mayr (1988) emphasized in his Toward a New Philosophy of Biology [that] recent progress in evolutionary biology has come mainly from conceptual clarification, not from improved measurements or better scientific laws" (Shrader-Frechette, this issue). We see an opportunity for philosophers of science, including philosophers of the special sciences, to develop more of these kinds of relationships with scientists doing socially relevant work, and also with policy makers and members of other stakeholder groups.

In addition to being consistent with some naturalized POS, SRPOS overlaps with much feminist philosophy of science and feminist epistemology. Feminist philosophy of science often deals with socially relevant topics, is highly interdisciplinary, has had an impact on scientific practice, and is concerned with the relationships between science and society at local, national, and global levels. So, as Richardson argues, feminist philosophy of science provides an excellent model for SRPOS. However, the marginalization of much feminist work within philosophy raises concerns about the degree of support that may be available for SRPOS itself - a topic to which we return in the next section.

\footnotetext{
6 There is a growing body of research on this topic [see, for example, Cartwright et al. 1996; Howard 2003, 2009; Kourany 2010; as well as Douglas (this issue)].
} 


\subsection{Is there an obligation to do SRPOS?}

As we have argued above, philosophers of science are in a good position to do work that can have a positive impact on public welfare, and such work is consistent with historical and contemporary practices in our discipline; but is there an obligation to do this sort of work? Some think that there is. Shrader-Frechette argues that philosophers have professional duties, and related duties as citizens, to use their training and expertise in ways that protect the public from science-related harms. She points to a variety of ways that philosophers of science can meet these obligations, which include doing SRPOS as part of one's research, teaching, and/or public service activities. Of course, the possibility of philosophers of science having a responsibility to do work that benefits the public merits a more careful and detailed treatment than can be offered here. However, by asking the question, 'why us?', philosophers of science might consider whether or not there are such responsibilities, what those responsibilities entail, and how we might best fulfill them. One possibility is to think of these responsibilities as ones that apply to and can be met by our discipline as a community as opposed to individual duties that all members must fulfill. In the next section, we reconceive our discipline as such a community and consider how that community can best support those doing SRPOS.

\section{How to support SRPOS: philosophy of science as an epistemic community}

Because of the wide range of benefits that can arise out of SRPOS, as well as possible responsibilities to have a positive impact on broader communities, philosophy of science would be well served by the production of more of this type of work. However, this imperative can raise a wide range of concerns, at least two of which include: (1) the possible conflict between obligations to do this sort of work and academic freedom, and (2) respecting the integrity and importance of a wide range of POS research programs, including those that don't fit within SRPOS and might be thought of as more mainstream investigations in the philosophy of science. In other words, how might philosophy of science reap the benefits and meet the possible responsibilities to do SRPOS while still respecting academic freedom and the existing range of philosophical work in the discipline? One way to ameliorate these tensions is to take a lesson from recent philosophical characterizations of science as a social practice (e.g., Longino 1990, 2001) in order to frame philosophy of science itself as a social practice.

\subsection{Reconceiving POS as an epistemic community}

The first move is to characterize POS itself as an epistemic, or knowledge-producing, community. It is easier to think of science than philosophy as a social activity. After all, much scientific research, with it laboratory research and fieldwork, involves a greater number and wider range of laborers. Also, there is a much higher incidence of collaborative research in many of the sciences than there is in philosophy. With online access to a library and a laptop computer, one can write a philosophy paper in relative academic isolation. However, when one considers the apprenticeship-like 
nature of philosophical training, the development of ideas through formal or informal social interactions (such as conferences), and the procedures of peer review, hiring and promotion, it is easy to see that the knowledge-producing practices and institutional structures of philosophy of science are highly social. In order for philosophical work to count as part of a productive career it must gain at least some support from other members of the philosophical community.

With this notion of philosophical community in mind, the number of ways that philosophers of science can benefit from SRPOS or fulfill a possible obligation to do SRPOS increases. An individual could devote part or all of her research efforts towards SRPOS. An individual could also support other community members who devote part or all of their research efforts toward SRPOS. If obligations to conduct SRPOS can be fulfilled by POS as a community, then there need not be a conflict with academic freedom nor with a healthy respect for other areas of research in philosophy of science. In fact, as we have illustrated above, there are benefits to both POS and SRPOS that arise from interactions between these research areas. In particular, some of the benefits of SRPOS include ways that it can contribute to mainstream POS, and SRPOS can at times draw upon more general POS scholarship. All that is called for is that one supports other community members who choose to engage in this sort of professional practice. It is important to note that a lack of support for SRPOS also impacts academic freedom - that of the scholars who wish to pursue this area of scholarship.

\subsection{Supporting SRPOS}

There are opportunities for POS to offer greater support to SRPOS. A striking aspect of the conversations at the both the APA mini-conference and PSA workshop that led to the development of this volume was the number of eminent philosophers of science who, even though they were enthusiastic about this project and had active research programs in SRPOS, reported that they either had concerns about or actively discouraged their graduate students from doing this work because they worried that it would have a negative impact on their students' employment prospects and career trajectories. Their sense was that philosophy of science and philosophy departments looking for new faculty tend to disincentivize this sort of work. This shows that rather than perceiving support for SRPOS, there is a group of faculty, including eminent philosophers from highly ranked departments, who perceive barriers. It is important both to respect the professional experience and expertise of these philosophers and to acknowledge that these reports provide local and anecdotal evidence. However, perceived barriers can become actual barriers because they can influence a wide range of professional practices, including decisions about whether or not to conduct this type of research, as well as the type of mentoring offered to those who are considering conducting this kind of research.

In addition to anecdotal evidence and perceptions of barriers that disincentivize SRPOS, there is scholarship regarding barriers that inhibit feminist SRPOS. In this issue, Sarah Richardson demonstrates the socially relevant nature of much feminist philosophy of science, as well as ways the culture of POS has impeded research in this area. She also shows how the marginalization and misrepresentation of feminist 
work has a negative impact on feminist philosophy of science. Additionally, Sally Haslanger (2008) has documented the striking under-representation of both women philosophers and feminist philosophy in top philosophy journals and raises significant questions regarding the review practices of some of these journals. Richardson's and Haslanger's work raises serious concerns about barriers erected within philosophy regarding feminist SRPOS. More generally, Douglas points out that the mission of the Philosophy of Science Association explicitly shifted away from SRPOS: in other words, the mandate to engage social issues disappeared. While there are areas within POS where one can expect to find SRPOS, opportunities remain for POS to better support and even encourage SRPOS.

Of course, POS cannot bear the burden of increasing the prevalence of SRPOS alone. There are multiple communities whose support or lack thereof can impact the prevalence of SRPOS. In addition to philosophy of science, relevant communities include people with other areas of philosophical specialization as well as philosophy departments themselves. Philosophy departments are especially important since they are the communities primarily responsible for hiring, promoting, and retaining philosophers of science. In most cases, a department that evaluates philosophy of science job candidates will include none or very few other philosophers of science. However, the involvement of other communities and the fact that it is largely non-POS philosophers who hire and promote philosophers of science does not remove agency from members of the philosophy of science community. There are important respects in which philosophers of science are still the gatekeepers- the committee members, letter writers, journal editors, conference organizers, and reviewers - who provide the professional credentials that hiring committees consider. Each of these gatekeeping practices represents an opportunity to give SRPOS concrete support.

However, these gatekeeping practices do not occur in isolation-they are part of the culture of POS. ${ }^{7}$ One can characterize the culture of an organization in terms of the interaction among norms, structures, and practices. ${ }^{8}$ In this context, norms refer to value judgments, structures to the ways that our work is organized, and practices to the everyday things that people say and do. Interactions among norms, practices, and structures can result in very resilient cultures. The culture of POS with regard to publishing and job success can be considered in this organizational theory context. Norms include judgments about what counts as central or excellent POS. Structures include the hierarchical organization of various journals, philosophy departments, and individual philosophers in terms of power and prestige. Practices are a very broad category; in this case, editorial and review practices as well as research practices are salient.

Culture surrounding professional publications is especially important. If SRPOS rarely appears in highly regarded philosophy journals, it signals and reinforces the devaluing of SRPOS. This structure and norm can influence a wide range of practices, including hiring practices and considerations of the prudence of engaging in SRPOS

\footnotetext{
7 This discussion need not assume that the culture of POS is homogeneous, just that there are common patterns within this community that tend to be self-reinforcing.

8 This sort of characterization of organizational culture is much discussed in sociology. For its development in and application to issues of gender and work, see, for example, Acker 1990; Kanter 1977; Hearn and Parkin 1983.
} 
as a career path. Consider editorial and review practices. An editor who has not developed a network of reviewers capable of assessing SRPOS, or a reviewer who gives knee-jerk dismissals of SRPOS work as 'not being philosophy', or as being 'merely applied philosophy', can have an impact that goes beyond that particular instance because such practices also influence the culture's structures and norms. Practices are the things we do and say. They are important not only in themselves, but because they are intertwined with structures and norms, which may then, in turn, reinforce a particular set of practices. If the culture of POS were supportive of SRPOS, one would find a strong representation of papers on this kind of research in top philosophy journals, those journals would cultivate a set of reviewers qualified to assess the merit of those papers, and they would have editorial and review practices that gave those papers serious consideration.

We are very grateful to those who have supported SRPOS by contributing to this special issue, including those who participated in the APA mini-conference and PSA workshop that led up to it, as well as the authors, reviewers, and editorial staff at Synthese, and especially John Symons, for their support of SRPOS as evidenced in this volume.

\section{Conclusion}

This paper provides an argument for a more socially relevant philosophy of science. Our aims in this paper are to characterize this body of work in philosophy of science, to argue for its importance, and to demonstrate that there are significant opportunities for POS to engage with and support this type of research. SRPOS is a highly pluralistic area which can include focusing on topics of scientific research that are relevant to public welfare, engaging with a wide range of stakeholder groups, and/or disseminating this philosophical work more broadly. The papers in this volume demonstrate both the breadth and depth of SRPOS, looking carefully at themes such as: developing collaborative relationships with scientists; addressing policy, regulation, and institutional structure; investigating the relationships among scientific and non-scientific communities; and assessing the effectiveness of the disciplinary norms, practices, and structures of philosophy of science itself. Pursuing this type of research can lead to benefits for various publics, for the practices and products of scientific research, and for philosophy itself. Furthermore, these benefits are often intertwined and mutually reinforcing; in many cases, the philosophical benefits that arise from SRPOS do so as a direct result of engaging with scientifically related social issues. Philosophy of science is in a particularly good position to do this type of work, as its members typically have the requisite skills, training, and education, and it is consistent with some historical and contemporary practices of our discipline. What we are calling for is the need to broaden our conception of our discipline not only to include this type of work, but also to support those who choose to pursue it. In order for SRPOS to thrive within our discipline, it must have the support of other philosophers of science who themselves may choose to pursue other, non-SRPOS areas of research. We hope that the arguments provided here for the importance of SRPOS to society and to our own discipline, in addition to the exemplars of such work provided in the rest of this 
volume, will lead to a more — and more highly valued—socially relevant philosophy of science.

Acknowledgements We are very grateful to the authors whose work appears in this volume and a conscientious and supportive community of reviewers who not only assessed but also offered constructive comments on many of these papers. We also extend our thanks to those who helped organize or gave papers at the APA mini-conference and PSA workshop that led to the production of this volume (Nancy Cartwright, Carl Cranor, Robert Crease, Heather Douglas, John Dupré, Sophia Efstathiou, Kevin Elliott, Lisa Gannett, Heidi Grasswick, Eric Martin, Susan Hawthorne, Don Howard, Janet Kourany, Lynn Hankinson Nelson, Julian Reiss, Sarah Richardson, Michael Root, Naomi Scheman, Kristin Shrader-Frechette, Anita Silvers, and Nancy Tuana), as well as to the audiences at these events for their lively and fruitful discussions. Finally, we are thankful for the organizational work of Sophia Efstathiou and the leadership of John Symons and the editorial staff at Synthese.

\section{References}

Acker, J. (1990). Hierarchies, jobs, bodies: A theory of gendered organizations. Gender \& Society, 4(2), 139-158.

Cartwright, N., Cat, J., Fleck, L., \& Uebel, T. (1996). Otto Neurath: Philosophy between science and politics. Cambridge: Cambridge University Press.

Fuller, S. (2008). Science studies goes public: A report on an ongoing performance. Spontaneous Generations: A Journal for the History and Philosophy of Science, 2(1), 11-21.

Giere, R. (2003). A new program for philosophy of science? Philosophy of Science, 70, 15-21.

Haslanger, S. (2008). Changing the ideology and culture of philosophy: Not by reason (alone). Hypatia, 23(2), 210-223.

Hearn, J., \& Parkin, W. (1983). Gender and organizations: A selective review and a critique of a neglected area. Organization Studies, 4(3), 219-242.

Howard, D. (2003). Two left turns make a right: On the curious political career of North American philosophy of science at midcentury. In A. Richardson \& G. Hardcastle (Eds.), Logical empiricism in North America, Minnesota studies in the philosophy of science (Vol XVIII) (pp. 25-93). Minneapolis: University of Minnesota Press.

Howard, D. (2009). Better red than dead-Putting an end to the social irrelevance of postwar philosophy of science. Science and Education, 18, 199-220.

Kanter, R. M. (1977). Men and women of the corporation. New York: Basic Books.

Keller, E. F. (1996). Feminism and science. In E. F. Keller \& H. E. Longino (Eds.), Feminism and science (pp. 28-40). New York: Oxford University Press.

Kourany, J. A. (2003a). A philosophy of science for the twenty-first century. Philosophy of Science, 70, $1-14$.

Kourany, J. A. (2003b). Reply to Giere. Philosophy of Science, 70, 22-26.

Kourany, J. A. (2010). Philosophy of science after feminism. New York: Oxford University Press.

Longino, H. E. (1990). Science as social knowledge: Values and objectivity in scientific inquiry. Princeton: Princeton University Press.

Longino, H. E. (2002). The fate of knowledge. Princeton: Princeton University Press. 